\title{
Performance Analysis of Iteratively Decoded Variable-Length Space-Time Coded Modulation
}

\author{
S. X. $\mathrm{Ng}^{1}$, W. Liu ${ }^{1}$, J. Wang ${ }^{1}$, M. Tao ${ }^{2}$, L.-L. Yang ${ }^{1}$ and L. Hanzo ${ }^{1}$ \\ ${ }^{1}$ School of ECS, University of Southampton, SO17 1BJ, United Kingdom. \\ Email: \{sxn,wl03r,jw02r,lly,lh\}@ecs.soton.ac.uk, http://www-mobile.ecs.soton.ac.uk \\ ${ }^{2}$ Department of Electrical and Computer Engineering, National University of Singapore, Singapore 117576. \\ Email: mxtao@nus.edu.sg
}

\begin{abstract}
It is demonstrated that iteratively Decoded Variable Length Space Time Coded Modulation (VL-STCM-ID) schemes are capable of simultaneously providing both coding gain as well as multiplexing and diversity gain. The VL-STCM-ID arrangement is a jointly designed iteratively decoded scheme combining source coding, channel coding, modulation as well as spatial diversity/multiplexing. In this contribution, we analyse the iterative decoding convergence of the VL-STCM-ID scheme using symbol-based three-dimensional EXIT charts. The performance of the VL-STCM-ID scheme is shown to be about $14.6 \mathrm{~dB}$ better than that of the Fixed Length STCM (FL-STCM) benchmarker at a source symbol error ratio of $10^{-4}$, when communicating over uncorrelated Rayleigh fading channels. The performance of the VL-STCM-ID scheme when communicating over correlated Rayleigh fading channels using imperfect channel state information is also studied.
\end{abstract}

\section{INTRODUCTION}

Shannon's separation theorem stated that source coding and channel coding is best carried out in isolation [1]. However, this theorem was formulated in the context of potentially infinite-delay, lossless entropy-coding and infinite block length channel coding. In practice, real-time wireless audio/video communications systems do not meet these ideal hypotheses. Specifically, the source encoded symbols often remain correlated, despite the lossy source encoder's efforts to remove all redundancy. Furthermore, they exhibit unequal error sensitivity. In these circumstances, it is often more efficient to use jointly designed source and channel encoders.

Space-time coding schemes, which employ multiple transmitters and receivers, are among the most efficient techniques designed for providing high data rates, which are capable of exploiting the high channel capacity potential of MultipleInput Multiple-Output (MIMO) channels [2]. More explicitly, Bell-lab's LAyered Space Time architecture (BLAST) [3] was designed for providing full-spatial-multiplexing gain, while Space Time Trellis Codes (STTC) [4] were designed for providing full-spatial-diversity gain.

A jointly designed source coding and Space Time Coded Modulation (STCM) scheme has been proposed in [5], [6]. This scheme employs novel two dimensional (2D) Variable Length Codes (VLCs) and is capable of exploiting both spatial and temporal domain diversity. More specifically, the number of activated transmit antennas equals the number of nonzero-energy symbols of the corresponding VLC codeword in

The financial support of the European Union under the auspices of the Newcom and Phoenix projects, as well as that of the EPSRC UK is gratefully acknowledged. the spatial domain, where each VLC codeword is transmitted during a single symbol period. Hence, the transmission frame length is determined by a fixed number of source symbols and therefore the proposed Variable Length STCM (VL-STCM) scheme does not incur synchronisation problems and does not require the transmission of side information. Additionally, the associated source correlation is converted into an increased minimum product distance [5], which leads to an increased coding gain.

For the sake of attaining additional iteration gains, the VLSTCM scheme was further developed in [7] by introducing parallel non-binary Unity-Rate Codes (URCs) between the variable-length space-time encoder and the modulator. The Iteratively Decoded (ID) VL-STCM (VL-STCM-ID) scheme achieves a significant coding/iteration gain over both the noniterative VL-STCM scheme and the Fixed Length STCM (FLSTCM) benchmarker [7]. The decoding convergence of the VL-STCM-ID scheme will be analysed and its performance using imperfect channel state information will be studied in this contribution.

\section{VL-STCM OVERVIEW}

Consider for example a source having $N_{s}=8$ possible discrete values and let the $l$ th value be represented by a symbol $s^{l}=l$ for $l \in\left\{1,2, \ldots, N_{s}\right\}$. We assume that the source symbols emitted are independent of each other and have unequal probabilities of occurrence given by

$$
P\left(s^{l+1}\right)=0.6 P\left(s^{l}\right)=0.6^{l} P\left(s^{1}\right),
$$

and $\sum_{l=1}^{N_{s}} P\left(s^{l}\right)=1$. Note that a source is correlated when its entropy rate $\mathcal{H}(s)$ is smaller than $\log _{2}\left(N_{s}\right)$ [8]. For the independent source considered, the source entropy rate equals the source entropy $H(s)$, which is given by: $\mathcal{H}(s)=H(s)=-\sum_{l=1}^{8} \log _{2}\left(P\left(s^{l}\right)\right) \cdot P\left(s^{l}\right)=2.302$ bit. Since $\mathcal{H}(s)<\log _{2}\left(N_{s}\right)$, the source considered is a correlated source, where the higher the source correlation the smaller the source entropy rate. Let us now consider a 2D VLC which encodes these $N_{s}=8$ possible source symbols using $N_{t}=3$ transmit antennas and BPSK modulation. The codebook can be formulated as a matrix:

$$
\mathbf{V}_{V L C}=\left[\begin{array}{llllllll}
\mathrm{x} & 1 & \mathrm{x} & 0 & \mathrm{x} & 0 & 1 & 1 \\
\mathrm{x} & \mathrm{x} & 0 & \mathrm{x} & 1 & 1 & 0 & 1 \\
0 & \mathrm{x} & \mathrm{x} & 1 & 1 & \mathrm{x} & 1 & 0
\end{array}\right],
$$

where each column corresponds to a specific VLC codeword conveying a particular source symbol. More specifically, each 


$$
\begin{array}{ccc} 
& A=\sqrt{\frac{N_{t}}{L_{\text {ave }}}} & \\
f(1)=-A & f(\mathrm{x})=0 & f(0)=A \\
\hdashline-A & 0 & A
\end{array}
$$

Fig. 1. The signal mapper of the VL-STCM.

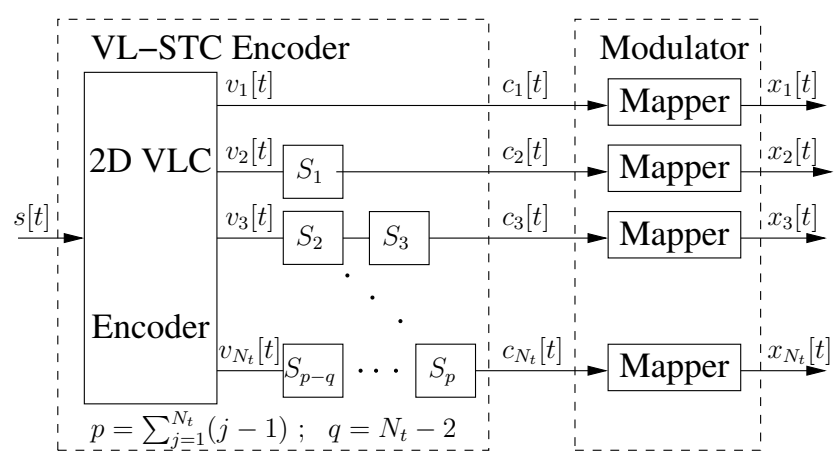

Fig. 2. Block diagram of the VL-STCM transmitter.

entry denoted as 0 and 1 represents the BPSK symbols to be transmitted, while ' $x$ ' corresponds to 'no transmission'. 'No transmission' implies that the corresponding transmit antenna sends no signal. Let the $l$ th source symbol $s^{l}$ be encoded using the $l$ th column of the $\mathbf{V}_{V L C}$ matrix seen in Equation 2. Hence, the source symbol $s^{1}$ is encoded into an $N_{t}$-element codeword using the first column of $\mathbf{V}_{V L C}$ in Equation 2, namely $\left[\begin{array}{lll}\mathrm{x} & \mathrm{x} & 0\end{array}\right]^{T}$, where the first and second transmit antennas are in the 'no transmission' mode, while the third antenna transmits an 'active' symbol represented by the binary value ' 0 '. Let $L\left(s^{l}\right)$ be the number of 'active' symbols in the VLC codeword assigned to source symbol $s^{l}$, then we may define the average codeword length of the 2D VLC as:

$$
L_{\text {ave }}=\sum_{l=1}^{N_{s}} P\left(s^{l}\right) L\left(s^{l}\right),
$$

where we have $L_{a v e}=1.233$ bit/VLC codeword for this system according to Equations 1 and 2. The corresponding BPSK signal mapper is characterised in Figure 1, where the 'no transmission' symbol is actually represented by the origin of the Euclidean space, i.e. we have $f(\mathrm{x})=0$, where $f($.$) is$ the mapping function. Since the 'no transmission' symbol is a zero energy symbol, the amount of energy saving can be computed from:

$$
A^{2}=\frac{N_{t}}{L_{\text {ave }}},
$$

where we have $A^{2}=3 / 1.233=2.433$, which is equivalent to $20 \log (A)=3.86 \mathrm{~dB}$. Hence, more transmitted energy is saved, when there are more 'no transmission' symbols in a VLC codeword. As a result, one can assign the more frequently occurring source symbols to the VLC codewords having more 'no transmission' components, in order to save transmit energy. The energy saved is then reallocated to the 'active' symbols for the sake of increasing their minimum Euclidean distance, as shown in Figure 1.
The 2D VLC matrix seen in Equation 2 was designed in [7], where $N_{t}=3$ transmit antennas were employed for transmitting the $N_{t}$-element 2D VLC codewords denoted as $\mathbf{v}=\left[v_{1} \ldots v_{N_{t}}\right]^{T}$ in Figure 2. It was shown in [7] that it attains a transmitter-diversity order quantified by the minimum Hamming distance of 2, a coding gain quantified by the minimum product distance of 5.92 and a spatial multiplexing gain quantified by $\log _{2}\left(N_{s} / M\right)=2$, where $M=2$ is the number of modulation levels of the original BPSK modulation and $N_{s}=8$ is the number of source symbols. The throughput of the scheme is given by $\eta=\log _{2}\left(N_{s}\right)=3 \mathrm{bit} / \mathrm{s} / \mathrm{Hz}$ and the Signal to Noise Ratio (SNR) per bit is given by $E_{b} / N_{0}=\gamma / \eta$, where $\gamma$ is the SNR per receive antenna.

The block diagram of the VL-STCM transmitter is illustrated in Figure 2, which can be represented by two fundamental blocks, namely the Variable Length Space Time Code (VL-STC) encoder and the modulator. As seen in Figure 2, a

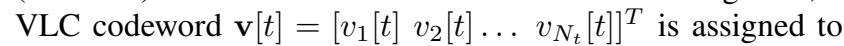
each of the source symbols $s[t]$ generated by the source at time instant $t$, where we have $s[t] \in\left\{1, \ldots, N_{s}\right\}$ and $N_{s}$ denotes the number of possible source symbols. Each of the VLC codewords $\mathbf{v}[t]$ seen in Figure 2 corresponds to one of the matrix columns in in Equation 2. As portrayed in Figure 2, the VLC codeword $\mathbf{v}[t]$ is transmitted diagonally across the space-time grid with the aid of shift registers denoted as $S_{k}$ in Figure 2, where we have $k \in\left\{1,2, \ldots, \sum_{j=1}^{N_{t}}(j-1)\right\}$. As we can see from Figure 2, the codeword $\mathbf{v}[t]=\left[v_{1}[t] v_{2}[t] \ldots v_{N_{t}}[t]\right]^{T}$ is transmitted using $N_{t}$ transmit antennas, where the $m$ th element of each VLC codeword, for $1 \leq m \leq N_{t}$, is delayed by $(m-1)$ shift register cells, before it is transmitted through the $m$ th transmit antenna. Hence, the $N_{t}$ number of components of each VLC codeword are transmitted on a diagonal of the space-time codeword matrix [5], [7].

\section{VL-STCM-ID OVERVIEW}

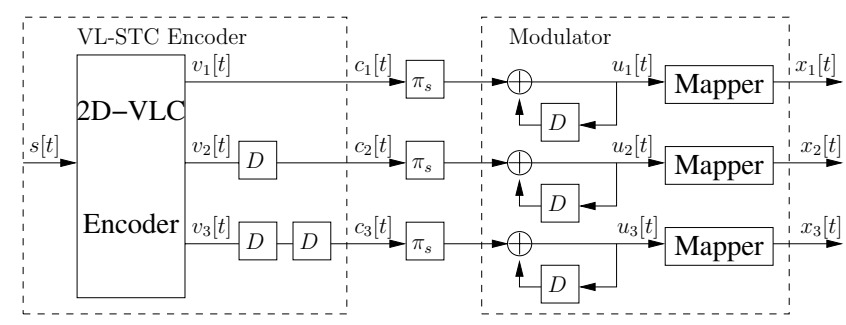

Fig. 3. The VL-STCM-ID transmitter employing $N_{t}=3$ transmit and $N_{r}=2$ receive antennas, where $\pi_{s}$ denotes symbol interleaver.

In order to invoke iterative detection and hence attain iteration gains as a benefit of the more meritoriously spread extrinsic information, a symbol-based random interleaver and a non-binary URC were introduced for each of the $N_{t}=3$ transmit antennas [7]. The $N_{t}=3$ parallel symbol-based interleavers were generated independently. As we can see from Figure 3 , the sequence corresponding to all the $m$ th elements in the space-time codeword $\left\{c_{m}[t]\right\}, m \in\{1,2,3\}$, is further interleaved and encoded by a non-binary URC, before being fed to the mapper and transmitted as $\left\{x_{m}[t]\right\}$. The non-binary URC employs a modulo- $\bar{M}$ adder, where again, $\bar{M}=3$ is the 
number of distinct symbols in the VLC space-time codeword and we have $c_{m}[t] \in\{0,1, \mathrm{x}\}$. Note that we represent the ' $\mathrm{x}$ ' symbol using the number ' 2 ' during the modulo- $\bar{M}$ addition.

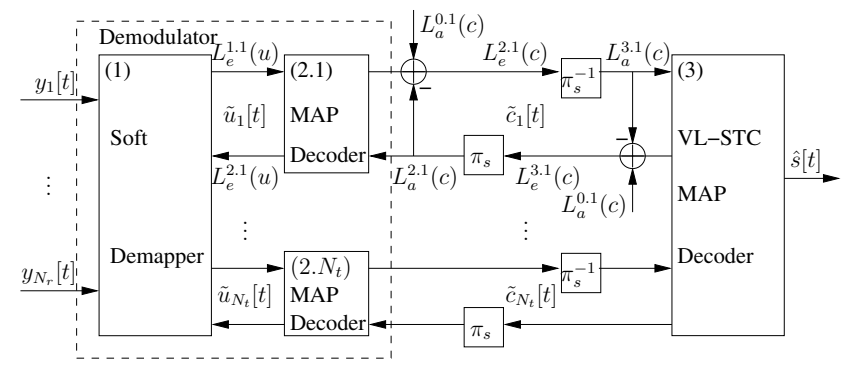

Fig. 4. The VL-STCM-ID receiver for an $N_{r} \times N_{t}$ MIMO system. The notation $($.$) and ($.$) indicates the extrinsicla priori probability and the$ hard decision estimate of $($.$) , respectively. The notation L_{(a, e)}^{i . m}(c, u)$ denotes the log-domain symbol probability of the VL-STC codeword $c$ or the URC codeword $u$ for the $m$ th transmitter. The subscripts $a$ and $e$ denote the a priori and extrinsic nature of the probabilities while the superscript $i . m$ identifies that the probabilities belong to the $i$ th stage decoder for the $m$ th transmitter. Note that $i=0$ means that the probabilities were calculated from the source symbol distribution.

At the receiver, the symbol-based log-domain MAP algorithm [9] is used by both the VL-STC decoder and the URC decoder. The block diagram of the VL-STCM-ID receiver is depicted in Figure 4, where we denote the log-domain symbol probability of the VL-STC codeword $c_{m}$ and the URC codeword $u_{m}$ for the $m$ th transmitter as $L_{(a, e)}^{i . m}(c)$ and $L_{(a, e)}^{i . m}(u)$, respectively. Furthermore, the subscripts $a$ and $e$ denote the a priori and extrinsic nature of the probabilities, while the superscript $i . m$ suggests that the probabilities belong to the $i$ th decoder stage of the $m$ th transmitter. Note that $i=0$ implies that the probabilities were calculated from the source symbol distribution. The extrinsic probability of the URC codeword of transmit antenna $m$, namely $P_{e}\left(u_{m}[t]\right)$, can be computed during each symbol period in the 'Soft Demapper' block of Figure 4. By dropping the time-related square bracket, we can compute $P_{e}\left(u_{m}\right)$ as:

$$
\begin{array}{r}
P_{e}\left(u_{m}=b\right)=\sum_{\substack{\mathbf{x} \in \chi(m, b) \\
\\
m \in\left\{1,2, \ldots, N_{t}\right\}, b \in\{0,1, \mathbf{x}\}}}\left(P(\mathbf{y} \mid \mathbf{x}) \prod_{\forall j \neq m}^{\text {all } j} P_{a}\left(u_{j}\right)\right),
\end{array}
$$

where the subset $\chi(m, b)$ contains all the phasor combinations for the transmitted signal vector $\mathbf{x}=\left[\begin{array}{llll}x_{1} & x_{2} & \ldots & x_{N_{t}}\end{array}\right]^{T}$ with $x_{m}=f\left(u_{m}=b\right)$, while $P(\mathbf{y} \mid \mathbf{x})$ is the conditional Probability Density Function (PDF) of the received signal. When communicating over Rayleigh fading MIMO channels, we have:

$$
P(\mathbf{y} \mid \mathbf{x})=\left(\frac{1}{2 \pi \sigma_{n}^{2}}\right)^{N_{r}} \exp \left(\frac{-\|\mathbf{y}-\mathbf{H} \mathbf{x}\|^{2}}{2 \sigma_{n}^{2}}\right),
$$

where $\sigma_{n}^{2}=N_{0} / 2$ is the noise variance, $\mathbf{y}$ is the $N_{r}$-element complex received signal vector and $\mathbf{H}$ is the $\left(N_{r} \times N_{t}\right)$ dimensional complex channel matrix during the time instant $t$. Furthermore, the a priori probability of $u_{m}$ in Equation 5 is computed from the extrinsic log-domain probability of the $m$ th URC MAP decoder as $P_{a}\left(u_{m}\right)=\exp \left(L_{e}^{2 . m}(u)\right)$, while the log-domain a priori probability of $u_{m}$ for the $m$ th URC MAP decoder is given by $L_{e}^{1 . m}(u)=\ln \left(P_{e}\left(u_{m}\right)\right)$.

It is possible to attain some a priori probability for the $N_{t}$-element VL-STC codewords, c, (which also constitute the URC's input words), given the source symbol occurrence probability specified in Equation 1. Explicitly, the probability of the $m$ th URC's input word $c_{m}$ can be expressed as:

$$
\begin{aligned}
P\left(c_{m}=d\right)= & \sum_{\substack{l \in \mu(m, d)\\
}} P\left(s^{l}\right), \\
& m \in\left\{1,2, \ldots, N_{t}\right\}, d \in\{0,1, \mathrm{x}\},
\end{aligned}
$$

where the subset $\mu(m, d)$ contains the specific indices of those columns in the VLC matrix, where the $m$ th row element in that column equals $d$. Hence, we have $L_{a}^{0 . m}(c)=\ln \left(P\left(c_{m}\right)\right)$ as an additional a priori probability for symbol $c_{m}$ during each iteration between the URC MAP decoder and the VLSTC MAP decoder, as shown in Figure 4. Note that $P\left(c_{m}\right)$ is directly computed from the source symbol occurrence probability $P\left(s^{l}\right)$, hence we do not use $P\left(s^{l}\right)$ again as the a priori probability of the VL-STC input word in the VL-STC MAP decoder, in order to avoid reusing the same information.

A full iteration consists of a soft demapper operation, $N_{t}=$ 3 URC MAP decoder operations and a VL-STC MAP decoder operation. For the non-iteratively decoded VL-STCM/FLSTCM, the soft demapper computes the a priori information

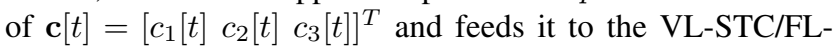
STCM MAP decoder. Note that the MAP decoder of VLSTCM/FL-STCM also benefits from the a priori probability of its input word $s[t]$. Hence, as the source becomes correlated, the VL-STCM-ID, VL-STCM and FL-STCM schemes will benefit from the a priori probability of the source symbols. However, FL-STCM attains no energy savings.

\section{Convergence AnAlysis}

Extrinsic Information Transfer (EXIT) charts designed for binary receivers [10] have been widely used for analysing the convergence behaviour of iterative decoding aided concatenated coding schemes. In this paper, we will employ the technique proposed in [11] for computing the non-binary EXIT functions. However, the convergence analysis of the proposed three-stage VL-STCM-ID scheme requires the employment of novel three Dimensional (3D) non-binary EXIT charts, which evolved from the binary 3D EXIT charts used in [12] for analysing multiple concatenated codes.

To elaborate a little further, EXIT charts visualise the input and output characteristics of the constituent MAP decoders in terms of the mutual information transfer between the input sequence and the a priori information at the input, as well as between the input sequence and the extrinsic information at the output of the constituent decoder. Hence, there are two steps in generating an EXIT chart. Firstly, we have to model the $a$ priori probabilities of the input sequence and then feed them to the decoder. Secondly, we have to compute the mutual information of the extrinsic probabilities at the output of the decoder. Let us now model the a priori probabilities of the VL-STC codeword, $\mathbf{c}=\left[\begin{array}{llll}c_{1} & c_{2} & \ldots & c_{N_{t}}\end{array}\right]^{T}$, where $c_{m}, m \in$ $\left\{1,2, \ldots, N_{t}\right\}$, is also the input symbol of the $m$ th URC. 
Let us denote the input symbol of the $m$ th URC as $c$, where the subscript $m$ is omitted for simplicity. Assume that the symbol $c$ is transmitted over an AWGN channel using the $\mathcal{M}=3$-phasor mapper shown in Figure 1 . The received signal is given by $y=x+n$, where $n$ is the AWGN noise having a zero mean and a variance of $\bar{\sigma}_{n}^{2}$. Furthermore, we have $x=f(c)$, where $f($.$) is the mapper function portrayed in$ Figure 1 . Since $f($.$) is a memoryless function, the probability$ of occurrence for $x$ is the same as that of $c$. Hence, we have $P(x)=P(c)$, which is expressed in Equation 7. At a given set of occurence probabilities for $x$, the mutual information between $x$ and $y$ can be formulated as:

$$
\begin{aligned}
I(x ; y) & =\sum_{i=1}^{\mathcal{M}} \int_{y} P\left(x_{i}, y\right) \log _{2}\left(\frac{P\left(x_{i}, y\right)}{P\left(x_{i}\right) P(y)}\right) d y \\
& =H(x)-H(x \mid y)
\end{aligned}
$$

where $H(x)$ is the entropy of $x$, given by:

$$
H(x)=-\sum_{i=1}^{\mathcal{M}} P\left(x_{i}\right) \log _{2}\left(P\left(x_{i}\right)\right),
$$

and $H(x \mid y)$ is the conditional entropy of $x$ given $y$, which can be expressed as:

$$
H(x \mid y)=\sum_{i=1}^{\mathcal{M}} P\left(x_{i}\right) E\left[\log _{2}\left(\sum_{j=1}^{\mathcal{M}} \frac{P\left(x_{j}\right)}{P\left(x_{i}\right)} \exp \left(\Psi_{i, j}\right)\right)\right]
$$

In Equation 10, we have $\exp \left(\Psi_{i, j}\right)=P\left(y \mid x_{j}\right) / P\left(y \mid x_{i}\right)$ and $P(y \mid x)$ is the conditional Gaussian PDF, while the exponent $\Psi_{i, j}$ is given by:

$$
\Psi_{i, j}=\frac{-\left|x_{i}-x_{j}+n\right|^{2}+|n|^{2}}{2 \bar{\sigma}_{n}^{2}} .
$$

The expectation term $E[$.$] in Equation 10$ is taken over the AWGN $n$. Hence, a curve can be generated for $I(x ; y)$ versus $\bar{\sigma}_{n}^{2}$, where the expectation term in Equation 10 is evaluated using Monte Carlo simulation. We can simplify Equation 8 to a form, where $I(x ; y)$ is expressed as a function of $\bar{\sigma}_{n}^{2}$. Let us denote this function as $J($.$) and we have I(x ; y)=J\left(\bar{\sigma}_{n}^{2}\right)$. Note that $I(x ; y)$ is monotonically decreasing with respect to $\bar{\sigma}_{n}^{2}$.

Let us now denote the a priori information of $c$ as $I_{A}(c)=$ $I(x ; y)$. At a given $I_{A}$ value we can find the corresponding noise variance with the aid of the inverse function $\bar{\sigma}_{n}^{2}=$ $J^{-1}\left(I_{A}(c)\right)$ using the $I(x ; y)$ versus $\bar{\sigma}_{n}^{2}$ curve. Then we can generate a noise sample $n^{\prime}$ having a variance of $\bar{\sigma}_{n}^{2}$. Consequently, we can produce $y^{\prime}=x+n^{\prime}$, where again $x=f(c)$ represents the mapper function portrayed in Figure 1 and $c$ is the actual input symbol of the $m$ th URC. Finally, we can generate the a priori symbol probabilities for $P_{a}(c)$ using the conditional Gaussian PDF:

$$
P_{a}(c)=\frac{1}{2 \pi \bar{\sigma}_{n}^{2}} \exp \left(\frac{-\left|y^{\prime}-f(c)\right|^{2}}{2 \bar{\sigma}_{n}^{2}}\right),
$$

for $c \in\{0,1, \mathrm{x}\}$. Then we feed these symbol probabilities to the corresponding MAP decoder. Note that the above method can be used for any symbol-interleaved serially concatenated coding schemes, where the symbol probabilities are directly created for a given $I_{A}$ value. The mutual information for the $N_{t}$-element VL-STC codeword $\mathbf{c}=\left[\begin{array}{llll}c_{1} & c_{2} & \ldots & c_{N_{t}}\end{array}\right]^{T}$ is the sum of the mutual information valid for its symbol components $c_{m}$, expressed as $I_{A}(\mathbf{c})=\sum_{m=1}^{N_{t}} I_{A}\left(c_{m}\right)$, where $I_{A}\left(c_{m}\right)$ is the mutual information of the $m$ th symbol component of the VL-STC codeword or the $m$ th URC's input symbol, given by Equation 8. Note that the maximum value of $I_{A}\left(c_{m}\right)$ equals the entropy of $c_{m}$ given by Equation 9.

Next, we compute the mutual information of the extrinsic symbol probabilities $I_{E}\left(c_{m}\right)$ at the output of the VL-STC or URC decoder for the symbol $c_{m}$ using the method proposed in [11]. Finally, the mutual information of the extrinsic symbol probabilities for the VL-STC codeword can be computed from $I_{E}(\mathbf{c})=\sum_{m=1}^{N_{t}} I_{E}\left(c_{m}\right)$. We also compute the mutual information for the URC codeword $\mathbf{u}$ based on the same procedure.

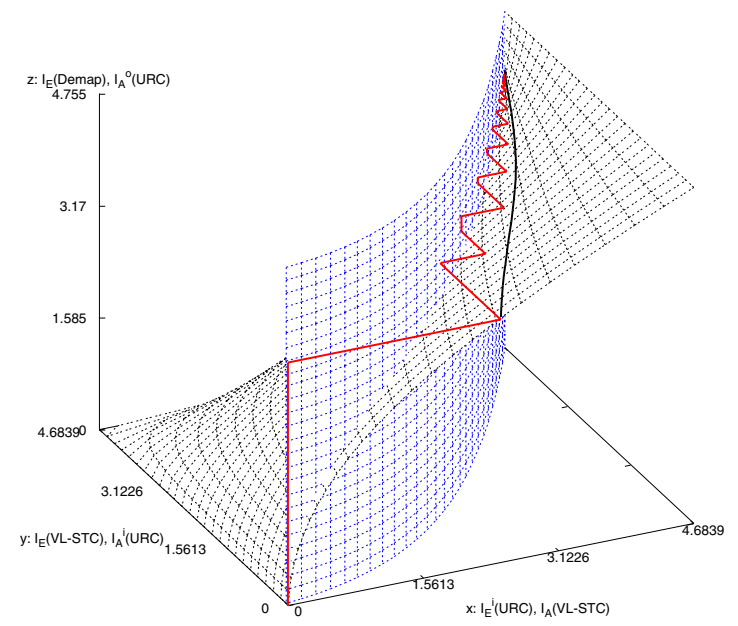

Fig. 5. The 3D EXIT charts for the VL-STCM-ID scheme having $N_{t}=3$ and $N_{r}=2$, when using an uncorrelated source. The iterative trajectory is computed at $E_{b} / N_{0}=4 \mathrm{~dB}$. The maximum value of an axis denotes the entropy of the corresponding symbol.

The 3D EXIT charts and the actual iterative decoding trajectories for the VL-STCM-ID scheme having $N_{t}=3$ and $N_{r}=2$ are shown in Figure 5 for an uncorrelated source. Let us denote the three axes of the 3D EXIT charts using the letters $\mathrm{x}, \mathrm{y}$ and $\mathrm{z}$, while $I_{A}\left(^{*}\right)$ and $I_{E}\left({ }^{*}\right)$ denote the a priori and extrinsic information for $(*)$, respectively, where $(*)$ is either the VL-STC MAP decoder (VL-STC) or the URC MAP decoder (URC) or, alternatively, the soft demapper (Demod). As we can see from Figure 4, each of the URC MAP decoders takes (provides) the a priori (extrinsic) probabilities of its input word $c$ and output word (or codeword) $u$ as the input (output). Hence, the mutual information of the input word and output word of the URC decoder will be represented by $I_{(A, E)}^{i}(\mathrm{URC})$ and $I_{(A, E)}^{o}(\mathrm{URC})$, respectively. Each of the $N_{t}$ symbol interleavers shown in Figures 3 has a length of 10000 symbols.

According to [12], the convergence between the soft demapper and the URC decoders can be represented by a curve drawn on the surface of the slanted EXIT plane in Figure 5, which is computed from the URC decoders' input word extrinsic probabilities. Furthermore, the intersection of the EXIT planes seen in Figure 5 represents the points of convergence between 


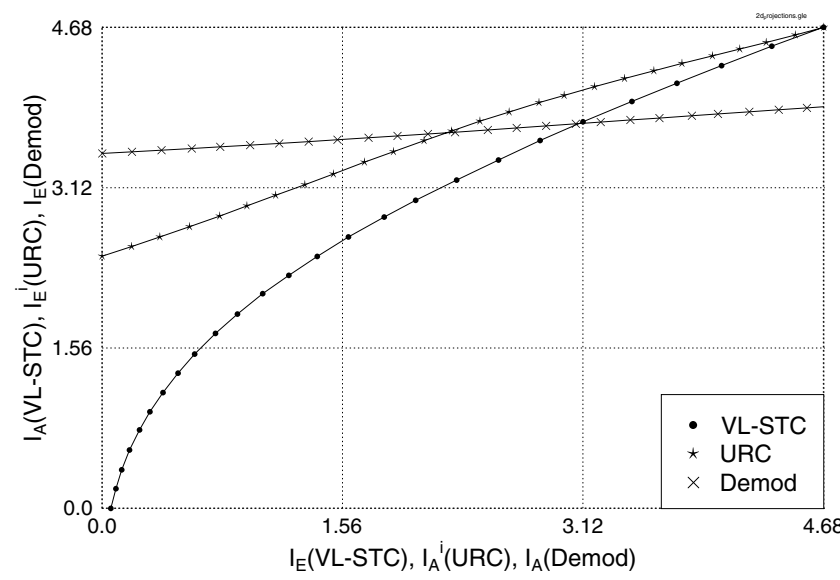

Fig. 6. The 2D EXIT charts projection for the VL-STCM-ID scheme having $N_{t}=3$ and $N_{r}=2$, when using an uncorrelated source at $E_{b} / N_{0}=4 \mathrm{~dB}$ The maximum value of an axis denotes the entropy of the corresponding symbol.

the URC decoder and the VL-STC decoder. Hence, by projecting these two convergence curves onto $\mathrm{z}=0$ in Figure 5 gives us the equivalent 2D EXIT chart seen in Figure 6. Therefore, the 3D EXIT charts generated for multiple concatenated codes can be projected onto an equivalent 2D EXIT chart [12].

More specifically, we can observe an open tunnel between the EXIT curves of the VL-STC and URC schemes in the 2D EXIT charts of Figure 6 at $E_{b} / N_{0}=4 \mathrm{~dB}$, which indicates that decoding convergence can be achieved. Note that the EXIT curve generated for the soft demapper is also depicted in Figure 6 at $E_{b} / N_{0}=4 \mathrm{~dB}$. This curve is almost flat and it intersects with the VL-STC EXIT curve, before the maximum value of 4.68 bits is reached. Hence, decoding convergence cannot be achieved at $E_{b} / N_{0}=4 \mathrm{~dB}$ when the URC was not invoked between the soft demapper and the VL-STC.

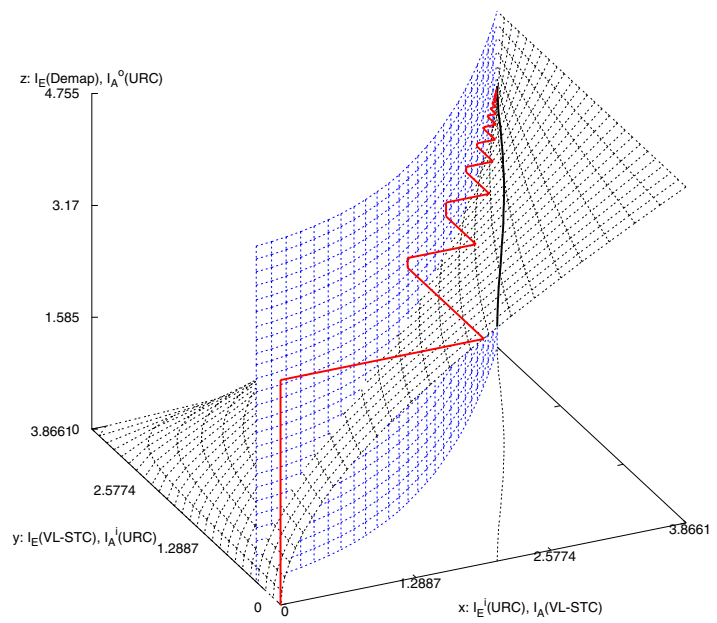

Fig. 7. The 3D EXIT charts for the VL-STCM-ID scheme having $N_{t}=3$ and $N_{r}=2$, when using the correlated source defined in Equation 1. The iterative trajectory is computed at $E_{b} / N_{0}=3 \mathrm{~dB}$. The maximum value of an axis denotes the entropy of the corresponding symbol.

Figure 7 shows the 3D EXIT charts and the corresponding convergence curve of the soft demapper and the URC decoder as well as the actual iterative decoding trajectory for the VLSTCM-ID scheme having $N_{t}=3$ and $N_{r}=2$ when using the correlated source defined in Equation 1. As the source becomes correlated, the entropy of the codeword $c_{m}$ for $m \in$ $\{1,2,3\}$ reduces. Hence, the maximum values for the $\mathrm{x}$ and $\mathrm{y}$ axes in Figure 7 are smaller than those in Figure 5. However, the open spatial segment of the 3D space between the two EXIT planes becomes wider, since the decoders exploit the additional a priori probabilities given by Equation 7, when the source is correlated. The convergence curve of the soft demapper and the URC decoder is projected as a dashed line onto $I_{E}($ Demod $)=0$ in Figure 7. Similarly, the projection of the intersection line between the VL-STC and URC EXIT planes is represented by the curve lying on the vertical EXIT plane at $I_{E}($ Demod $)=0$. As can be seen from Figure 7 at $I_{E}($ Demod $)=0$, an open tunnel exists between the two projection curves at $E_{b} / N_{0}=3 \mathrm{~dB}$. Hence, the iterative decoder converged at $E_{b} / N_{0}=3 \mathrm{~dB}$, i.e. at a $1 \mathrm{~dB}$ lower value, when employing the correlated source instead of the uncorrelated source.

According to the MIMO channel capacity formula derived for the Discrete-Input Continuous-Output Memoryless Channel (DCMC) in [13], the DCMC capacity for the $N_{r}=2$ and $N_{t}=3$ MIMO scheme employing the signal mapper seen in Figure 1 is $E_{b} / N_{0}=1.25 \mathrm{~dB}$ at a bandwidth efficiency of $3 \mathrm{bit} / \mathrm{s} / \mathrm{Hz}$. Hence, the performance of the VL-STCM-ID schemes in Figures 5 and 7 is about $2.75 \mathrm{~dB}$ and $1.75 \mathrm{~dB}$ away from the MIMO channel capacity.

\section{Simulation RESUlts}

The Fixed Length (FL) STCM (FL-STCM) scheme used in [7] employed the following FL Codebook (FLC) matrix:

$$
\mathbf{V}_{F L C}=\left[\begin{array}{llllllll}
0 & 0 & 0 & 0 & 1 & 1 & 1 & 1 \\
0 & 0 & 1 & 1 & 0 & 0 & 1 & 1 \\
0 & 1 & 0 & 1 & 0 & 1 & 0 & 1
\end{array}\right]
$$

The FL-STCM transmitter obeys the schematic of Figure 2, except that it employs the $\mathbf{V}_{F L C}$ of Equation 13. For the FLSTCM, the minimum Hamming distance and product distance are 1 and 4, respectively. It attains the same multiplexing gain as that of the VL-STCM or VL-STCM-ID arrangements. Note that it is possible to create an iterative FL-STCM-ID scheme by replacing the VL-STC encoder in Figure 3 with the FLSTC encoder. However, the EXIT curve of the FL-STC scheme of Equation 13 was found to be too flat for attaining any iteration gain due to its unity minimum Hamming distance. Let us now evaluate the performance of the VL-STCM, VLSTCM-ID and FL-STCM schemes in terms of their source Symbol Error Ratio (SER) versus the $E_{b} / N_{0}$ ratio. Again we have $E_{b} / N_{0}=\gamma / \eta$, where $\gamma$ is the SNR per receive antenna and $\eta=\log _{2}\left(N_{s}\right)=3 \mathrm{bit} / \mathrm{s} / \mathrm{Hz}$ is the effective information throughput. Throughout our simulations, we use three transmit antennas and two receive antennas. The interleaver length is set to 10000 symbols.

Figure 8 depicts the SER versus $E_{b} / N_{0}$ performance of the VL-STCM, VL-STCM-ID and FL-STCM schemes when communicating over uncorrelated Rayleigh fading channels. As expected, the VL-STCM arrangement attains a higher gain when the source is correlated compared to FL-STCM. However, the FL-STCM benchmarker also benefits from the probability-related a priori information of the source symbols, 


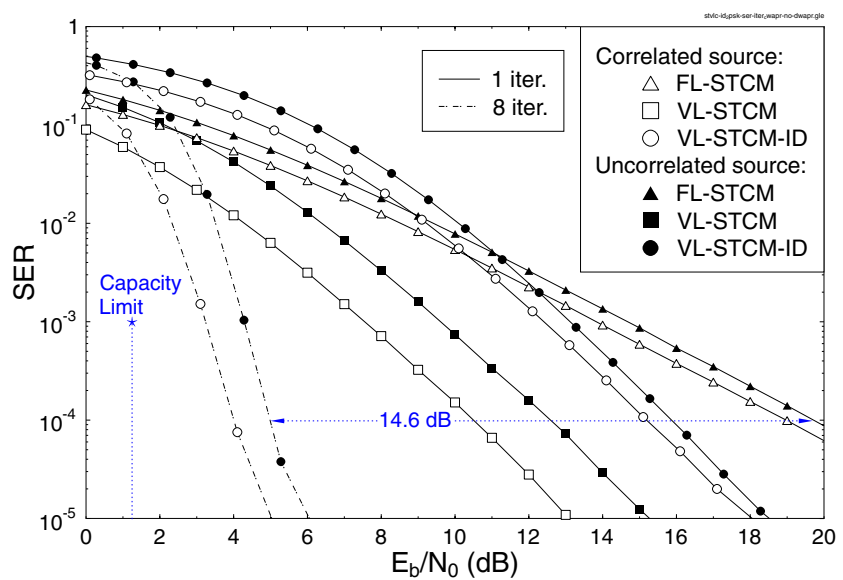

Fig. 8. SER versus $E_{b} / N_{0}$ performance of the VL-STCM, VL-STCMID and FL-STCM schemes, when communicating over uncorrelated Rayleigh fading channels using BPSK, $N_{t}=3$ and $N_{r}=2$.

as the source becomes correlated. On the other hand, the coding gain attained as a benefit of transmitting correlated source symbols increases, as the number of iterations invoked by the VL-STCM-ID scheme increases. The performance of VL-STCM-ID at SER $=10^{-4}$ after the 8th iteration is approximately 6.5 (15) $\mathrm{dB}$ and 7.5 (14.6) $\mathrm{dB}$ better than that of the VL-STCM (FL-STCM) scheme, when employing correlated and uncorrelated sources, respectively.

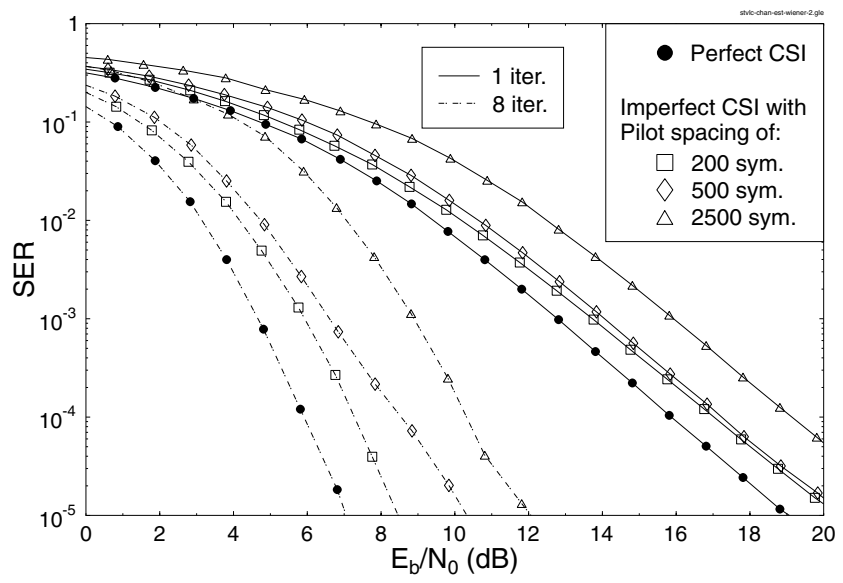

Fig. 9. SER versus $E_{b} / N_{0}$ performance of the VL-STCM, VL-STCMID and FL-STCM schemes, when communicating over correlated Rayleigh fading channels at a normalised Doppler frequency of $10^{-4}$, using Wiener filter based channel estimation, BPSK, $N_{t}=3$ and $N_{r}=2$.

Figure 9 shows the SER versus $E_{b} / N_{0}$ performance of the VL-STCM-ID scheme, when communicating over correlated Rayleigh fading channels at a normalised Doppler frequency of $10^{-4}$ and using Wiener filter based channel estimation [14]. The source is correlated, as formulated in Equation 1. As we can see from Figure 9, when the Channel State Information (CSI) becomes more accurate as a benefit of using a shorter pilot symbol spacing, the performance of the VL-STCM-ID approaches that of the ideal case with perfect CSI. When using a pilot spacing of 200 symbols, there are 10000/200 $=50$ subframes within the 10000-symbol frame. Note that $N_{t}$ frames are transmitted in parallel from $N_{t}$ transmit antennas in order to convey a space-time coded frame in the MIMO system.
In order to detect the channel coefficients from each transmit antenna, $N_{t}$ number of orthogonal pilot sequences are inserted at the end of the $N_{t}$ sub-frames transmitted in parallel. We need a minimum of $N_{t}$ pilot symbols for each of the pilot sequences in order to construct $N_{t}$ orthogonal pilot sequences. Hence, when using a pilot spacing of 200 symbols, $N_{t}=3$ pilot symbols are inserted for every 200 VL-STCM-ID coded symbols, which corresponds to a transmission overhead of $3 / 200=1.5 \%$ and requires inserting $\left(10000 \times N_{t} / 200\right)=150$ pilot symbols into the transmission frame of $10000+150=$ 10150 symbols.

\section{CONCLUSiOnS}

The convergence properties of the VL-STCM-ID scheme were analysed using EXIT charts. A significant iteration gain was achieved by the VL-STCM-ID scheme, which hence outperformed both the non-iterative VL-STCM scheme as well as the FL-STCM benchmarker with the aid of $N_{t}$ number of unity-rate recursive precoders. The near capacity performance of VL-STCM-ID scheme is retained, when employing a realistic Wiener filter based channel estimator, rather than using perfect channel estimation.

\section{REFERENCES}

[1] C. Shannon, Mathematical Theory of Communication. University of Illinois Press, 1963.

[2] E. Telatar, "Capacity of multi-antenna Gaussian channels," European Transactions on Telecommunication, vol. 10, pp. 585-595, Nov-Dec 1999.

[3] G. J. Foschini, Jr., "Layered space-time architecture for wireless communication in a fading environment when using multi-element antennas," Bell Labs Technical Journal, pp. 41-59, 1996.

[4] V. Tarokh, N. Seshadri and A. R. Calderbank, "Space-time codes for high rate wireless communication: Performance analysis and code construction," IEEE Transactions on Information Theory, vol. 44, pp. 744-765, March 1998.

[5] S. X. Ng, J. Wang, L.-L. Yang and L. Hanzo, "Variable Length Space Time Coded Modulation," in IEEE Vehicular Technology Conference, (Dallas, Texas), pp. 1049-1053, 25-28 September 2005.

[6] M. Tao and S. X. Ng, "Space Time Variable Length Coding for Wireless Multimedia Communications," in Proceedings of 3rd International Conference on Communications, Circuits and Systems (ICCCAS), (Hong Kong, China), pp. 51-55, 27-30 May 2005.

[7] S. X. Ng and L. Hanzo, "Iteratively Decoded Variable Length Space Time Coded Modulation," in Proceedings of IEE International Symposium on Communication Theory \& Applications (ISCTA), (Ambleside, Lake District, UK), pp. 309-314, 17 - 22 July 2005.

[8] T. M. Cover and J. A. Thomas, Elements of Information Theory. New York, USA: John Wiley IEEE Press, 1991.

[9] L. Hanzo, T. H. Liew and B. L. Yeap, Turbo Coding, Turbo Equalisation and Space Time Coding for Transmission over Wireless channels. New York, USA: John Wiley IEEE Press, 2002.

[10] S. ten Brink, "Convergence behaviour of iteratively decoded parallel concatenated codes," IEEE Transactions on Communications, vol. 49, pp. 1727-1737, October 2001.

[11] J. Kliewer, S. X. Ng and L. Hanzo, "On the computation of EXIT characteristics for symbol-based iterative decoding," in 4th International Symposium on Turbo Codes in connection with 6th International ITGConference on Source and Channel Coding, (Munich, Germany), 3-7 April 2006

[12] F. Brannstrom, L. K. Rasmussen and A. J. Grant, "Convergence analysis and optimal scheduling for multiple concatenated codes," IEEE Transaction on Information Theory, pp. 3354-3364, Sept 2005.

[13] S. X. Ng and Hanzo, "On the MIMO Channel Capacity of MultiDimensional Signal Sets," IEEE Transactions on Vehicular Technology, vol. 55, pp. 528-536, March 2006.

[14] J. K. Cavers, "An Analysis of Pilot Symbol Assistes Modulation for Rayleigh Fading Channels," IEEE Transactions on Vehicular Technology, vol. 40, pp. 686-693, November 1991. 